Journal of Organometallic Chemistry, 388 (1990) 203-214

Elsevier Sequoia S.A., Lausanne - Printed in The Netherlands

JOM 20666

\title{
Reaktivitätsstudien an S- und Se-verbrückten zweikernigen Carbonyl-Rhodium-Komplexen
}

\author{
Henri Brunner, Norbert Janietz, Joachim Wachter *, \\ Institut für Anorganische Chemie der Universität Regensburg, Universitätsstrasse 31, \\ D-8400 Regensburg (B.R.D.)
}

\author{
Hans-Peter Neumann, Bernd Nuber und Manfred L. Ziegler \\ Anorganisch-Chemisches Institut der Universität Heidelberg, Im Neuenheimer Feld 270, \\ D-6900 Heidelberg (B.R.D.)
}

(Eingegangen den 14. Dezember 1989)

\begin{abstract}
The reactivity of singly and doubly selenium-bridged complexes containing two $\mathrm{Cp}^{\star} \mathrm{Rh}(\mathrm{CO})$ fragments $\left(\mathrm{Cp}^{\star}=\eta^{5}-\mathrm{C}_{5} \mathrm{Me}_{5}\right)$ has been investigated under various conditions. The addition of $\mathrm{SO}_{3}$ to $\mathrm{Cp}^{\star}{ }_{2} \mathrm{Rh}_{2}(\mathrm{CO})_{2}(\mu$-Se) gives a novel $\mu$-SeSO ligand, whose structure was determined by an X-ray diffraction study. The complexes $\mathrm{Cp}^{\star}{ }_{2} \mathrm{Rh}_{2}(\mathrm{CO})_{2}(\mu-\mathrm{E})_{2}(\mathrm{E}=\mathrm{S}$, Se) undergo a disproportionation reaction in solution: the clusters $\mathrm{Cp}^{\star}{ }_{3} \mathrm{Rh}_{3}\left(\mu_{3}-\mathrm{CO}\right)\left(\mu_{3}-\mathrm{E}\right)$ were isolated and structurally characterized, whereas the remaining soluble, presumably mononuclear species was only detected by IR spectroscopy. In contrast, $\mathrm{Cp}^{\star}{ }_{2} \mathrm{Rh}_{2}(\mathrm{CO})_{2}(\mu \text {-Se) })_{2}$ dimerizes upon warming to give $\mathrm{Cp}^{\star}{ }_{4} \mathrm{Rh}_{4}\left(\mu_{3}-\mathrm{Se}\right)_{4}$ with the loss of its $\mathrm{CO}$ groups. The 72-e heterocubane clusters $\mathrm{Cp}_{4}^{\star} \mathrm{Co}_{2} \mathrm{Rh}_{2} \mathrm{Sc}_{4}$ and $\mathrm{Cp}_{4}{ }_{4} \mathrm{Co}_{4} \mathrm{Se}_{4}$ have becn synthesized from $\mathrm{Cp}_{2}{ }_{2} \mathrm{CoM}(\mathrm{CO})_{2} \mathrm{Se}_{2}(\mathrm{M}=\mathrm{Co}, \mathrm{Rh})$. The cluster $\mathrm{Cp}^{\star}{ }_{3} \mathrm{Rh}_{3}(\mathrm{CO})_{3}$ was isolated as a further byproduct from the synthesis of $\mathrm{Cp}^{\star}{ }_{2} \mathrm{Rh}_{2}(\mathrm{CO})_{2}$. The IR spectra and the $\mathrm{X}$-ray crystallographic study show that the complex contains one $\mu_{3}$-CO and two $\mu_{2}-\mathrm{CO}$ ligands.
\end{abstract}

\section{Zusammenfassung}

Es wurde die Reaktivität von einfach und doppelt selenverbrückten Komplexen mit $\mathrm{Cp}^{\star} \mathrm{Rh}(\mathrm{CO})$-Fragmenten $\left(\mathrm{Cp}^{\star}=\eta^{5}-\mathrm{C}_{5} \mathrm{Me}_{5}\right)$ unter verschiedenen Gesichtspunkten untersucht. Die Addition von $\mathrm{SO}_{3}$ an $\mathrm{Cp}^{\star}{ }_{2} \mathrm{Rh}_{2}(\mathrm{CO})_{2}(\mu$-Se) verläuft unter Bildung eines neuartigen $\mu$-SeSO ${ }_{3}$-Liganden, dessen Struktur durch Röntgenstrukturanalyse bestimmt wurde. Die Komplexe $\mathrm{Cp}^{\star}{ }_{2} \mathrm{Rh}_{2}(\mathrm{CO})_{2}(\mu-\mathrm{E})_{2}(\mathrm{E}=\mathrm{S}$, Se) unterliegen in Lösung einer Disproportionierungsreaktion: Die Cluster $\mathrm{Cp}^{\star}{ }_{3} \mathrm{Rh}_{3}\left(\mu_{3^{-}}\right.$ $\mathrm{CO})\left(\mu_{3}-\mathrm{E}\right)$ können isoliert und strukturell charakterisiert werden, während eine in 
Lösung verbleibende, vermutlich einkernige Spezies nur IR-spektroskopisch erfassbar war. $\mathrm{Cp}_{2}{ }_{2} \mathrm{Rh}_{2}(\mathrm{CO})_{2}(\mu \text {-Se })_{2}$ dimerisiert dagegen in der Wärme unter Verlust seiner CO-Gruppen zu $\mathrm{Cp}^{\star}{ }_{4} \mathrm{Rh}_{4}\left(\mu_{3}-\mathrm{Se}\right)_{4}$. Durch Ersatz von $\mathrm{Rh}$ durch Co können die 72e-Heterocuban-Cluster $\mathrm{Cp}^{\star}{ }_{4} \mathrm{Co}_{2} \mathrm{Rh}_{2} \mathrm{Se}_{4}$ und $\mathrm{Cp}^{\star}{ }_{4} \mathrm{Co}_{4} \mathrm{Se}_{4}$ synthetisiert werden. Als weiteres Nebenprodukt bei der Synthese von $\mathrm{Cp}^{\star}{ }_{2} \mathrm{Rh}_{2}(\mathrm{CO})_{2}$ konnte $\mathrm{Cp}_{3}^{\star} \mathrm{Rh}_{3}(\mathrm{CO})_{3}$ isoliert werden. Der Komplex besitzt laut IR-Spektren und Röntgenstrukturanalyse einen $\mu_{3}$-CO- und zwei $\mu_{2}$-CO-Liganden.

\section{Einleitung}

Die Reaktion von $\mathrm{Cp}^{\star}{ }_{2} \mathrm{Rh}_{2}(\mu-\mathrm{CO})_{2}\left(\mathrm{Cp}^{\star}=\eta^{5}-\mathrm{C}_{5} \mathrm{Me}_{5}\right)$ mit elementarem Schwefel oder Selen führt zunächst zu den chalkogenverbrückten, noch CO-haltigen Komplexen 1a, b und 2 [1]. Diese Produkte reagieren mit überschüssigem Chalkogen zu den CO-freien Verbindungen $\mathrm{Cp}_{2}^{\star} \mathrm{Rh}_{2} \mathrm{~S}_{8}$ [2] und $\mathrm{Cp}_{2}^{\star}{ }_{2} \mathrm{Rh}_{2} \mathrm{Se}_{5}$ [3], deren Strukturen bereits Gegenstand umfangreicher Untersuchungen waren. In ersten Untersuchungen zum Aufbau derartiger chalkogenreicher Zweikernkomplexe konnte gezeigt werden, dass nicht nur die CO-Liganden in $\mathbf{I}$ und $\mathbf{2}$ substitutionslabil sind, sondern auch die Chalkogenbrücken [1b]. In Fortführung dieser Arbeiten wurde nunmehr versucht, die Reaktivität von 1 und 2 zur Synthese neuer Rhodiumchalkogenkomplexe zu nutzen.

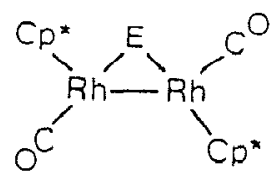

$(1 a, b)$

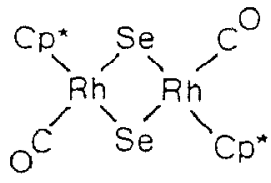

(2)

$(a: E=S ; b: E=S e)$

Die Reaktion von $\mathrm{Cp}_{2}{ }_{2} \mathrm{Rh}_{2}(\mathrm{CO})_{2} \mathrm{Se}(1 \mathrm{~b})$ mit $\mathrm{SO}_{2}$ bzw. $\mathrm{SO}_{3}$

Für die Reaktion von $1 \mathrm{~b}$ mit $\mathrm{SO}_{2}$ bieten sich mindestens drei verschiedene Reaktionsmöglichkeiten an, die u. a. auch aus seiner Isolobalbeziehung zu $\mathrm{Cp}_{2} \mathrm{Rh}_{2}(\mathrm{CO})_{2}\left(\mu-\mathrm{CH}_{2}\right)$ und dessen Reaktionen mit $\mathrm{SO}_{2}$ [1a] abgeleitet werden: (a) Einschub von $\mathrm{SO}_{2}$ in die Rh-Rh-Bindung unter Ausbildung eines Vierrings; (b) Substitution des Se-Liganden durch das isoelektronische $\mathrm{SO}_{2}$; (c) Nutzung der freien Elektronenpaare der Se-Brücke unter Ausbildung eines Lewissäureaddukts [4].

Leitet man durch die Lösung von $1 \mathrm{~b}$ in Toluol/Pentan einen kräftigen $\mathrm{SO}_{2}$-Strom, so bildet sich ein Produktgemisch, das nicht getrennt werden kann. IR-spektroskopisch kann aus einer $\nu(\mathrm{CO})$-Frequenz bei $1985 \mathrm{~cm}^{-1}(\mathrm{KBr})$ auf das Vorliegen von $\mathrm{Cp}^{\star}{ }_{2} \mathrm{Rh}_{2}(\mathrm{CO})_{2}\left(\mu-\mathrm{SO}_{2}\right)$ [1a] geschlossen werden, eine weitere $\mathrm{CO}$-Absorption bei $2030 \mathrm{~cm}^{-1}$ kann nicht schlüssig interpretiert werden. 
$\mathrm{lb}+\mathrm{SO}_{3} \longrightarrow \overbrace{\mathrm{O}^{\mathrm{C}^{\prime}}}^{\mathrm{Rh}^{*}} \mathrm{Se}_{\mathrm{Rh}^{\mathrm{O}}}^{\mathrm{SO}_{3}} \mathrm{C}^{\mathrm{O}}$

(3)

Setzt man 1b mit einer äquimolaren Menge an Pyridin $\cdot \mathrm{SO}_{3} \mathrm{um}$, so entsteht in 82\% Ausbeute der dunkelviolette Komplex 3 (Gl. 1). Im IR-Spektrum von 3 ist eine deutliche Verschiebung der CO-Absorption zu höheren Wellenzahlen im Vergleich zum Edukt zu beobachten ( $\nu(C O): 3,1992 ; 1 b, 1953 \mathrm{~cm}^{-1}$ ). Dies ist vereinbar mit den gegenüber dem $\mu$-Se-Liganden stärkeren $\pi$-Akzeptoreigenschaften des $\mu$ $\mathrm{SeSO}_{3}$-Liganden. Die $\mathrm{SO}_{3}$-Gruppierung gibt sich durch drei sehr starke $\nu$ (SO)Frequenzen bei 1239,1230 und $593 \mathrm{~cm}^{-1}$ zu erkennen. Charakteristisch ist auch die thermische Empfindlichkeit von 3 unter den Bedingungen des FD-Massenspektrums, unter denen sich 3 zersetzt und lediglich eine Vielzahl von Folgeprodukten liefert. Ähnliches Verhalten zeigen Komplexe des Typs $\mathrm{Cp}_{2}^{\star}{ }_{2} \mathrm{M}_{2} \mathrm{~S}_{n} \cdot \mathrm{SO}_{3}(\mathrm{M}=\mathrm{Cr}$, Mo; $n=4,5$ ), in denen $\mathrm{SO}_{3}$ an Brückensulfidliganden koordiniert ist [5].

Eine an einem Einkristall von 3 durchgeführte Röntgenstrukturanalyse (Tab. 1) zeigt analog zum Edukt 1b [1b] einen $\mathrm{Rh}_{2}$ Se-Dreiring als tragendes Strukturelement

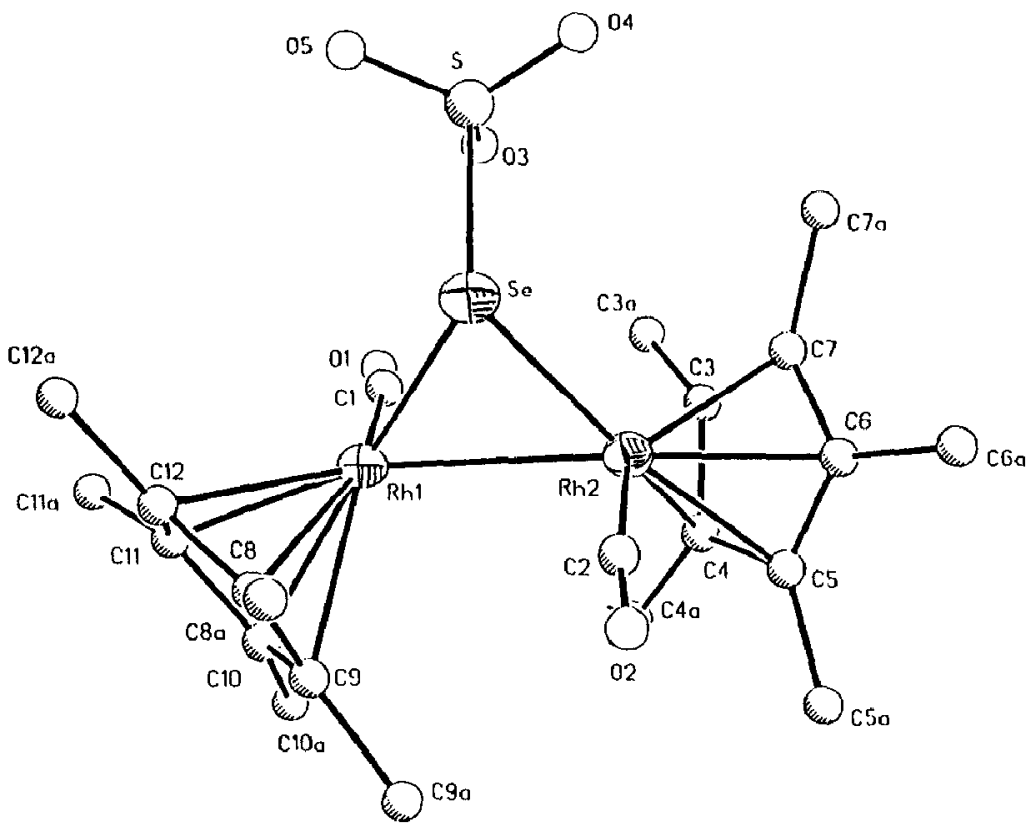

Fig. 1. Struktur von $\mathrm{Cp}_{2}^{\star}{ }_{2} \mathrm{Rh}_{2}(\mathrm{CO})_{2}\left(\mu-\mathrm{SeSO}_{3}\right)(3)$ Kristall. Ausgewählte Bindungslängen ( $\left.\mathrm{A}\right)$ und -winkel $\left({ }^{\circ}\right): \operatorname{Rh}(1)-\operatorname{Rh}(2)$ 2.769(2), $\mathrm{Rh}(1)-\mathrm{Se} 2.417(2), \mathrm{Rh}(1)-\mathrm{C}(1)$ 1.89(2), $\mathrm{Rh}(1)-\mathrm{C}(\mathrm{Cp} 1)_{\text {Mittel }} 2.26(3), \mathrm{Rh}(1)-$ $\mathrm{Cp}(1)_{\text {Zentr. }} 1.899(2), \mathrm{Rh}(2)-\mathrm{Se} 2.440(3), \mathrm{Rh}(2)-\mathrm{C}(2) 1.93(2), \mathrm{Rh}(2)-\mathrm{C}(\mathrm{Cp} 2)_{\text {Mittel }} 2.27(3), \mathrm{Rh}(2)-\mathrm{Cp}(2)_{\text {Zentr. }}$ 1.919(2), S-Se 2.301(6); Rh(2)-Rh(1)-C(1) 90.6(7), Se-Rh(1)-C(1) 97.6(8), $R h(2)-R h(1)-S e$ 55.6(1), Rh(1)-Se-Rh(2) 69.5(1), Rh(1)-Rh(2)-C(2) 85.1(6), Cp(1) zentr- - Rh(1)-Se 130.9(1), Cp(2) Zentr. - Rh(2)-Se 142.1(1). 
Tabelle 1

Atomkoordinaten und Temperaturfaktoren von $\mathrm{Cp}_{2}^{\star}{ }_{2} \mathrm{Rh}_{2}(\mathrm{CO})_{2}\left(\mu-\mathrm{SeSO}_{3}\right)(3)$

\begin{tabular}{|c|c|c|c|c|}
\hline Atom & $x$ & $y$ & $z$ & $U$ \\
\hline$\overline{\operatorname{Rh}(1)}$ & $0.7604(1)$ & $0.9235(1)$ & $0.8866(1)$ & $0.025(1)^{a}$ \\
\hline $\operatorname{Rh}(2)$ & $0.7019(1)$ & $1.1128(1)$ & $0.8745(1)$ & $0.025(1)^{a}$ \\
\hline $\mathrm{Se}$ & $0.7205(2)$ & $1.0262(1)$ & $0.9930(1)$ & $0.030(1)^{\circ}$ \\
\hline $\mathrm{S}$ & $0.5127(6)$ & $0.9965(4)$ & $1.0465(3)$ & $0.035(2)$ \\
\hline$O(1)$ & $0,481(2)$ & $0.860(1)$ & $0.840(1)$ & $0.057(5)$ \\
\hline$O(2)$ & $0.997(1)$ & $1.166(1)$ & $0.891(1)$ & $0.048(4)$ \\
\hline$O(3)$ & $0.421(1)$ & $0.970(1)$ & $0.987(1)$ & $0.040(4)$ \\
\hline$O(4)$ & $0.483(1)$ & $1.082(1)$ & $1.083(1)$ & $0.056(5)$ \\
\hline$O(5)$ & $0.545(1)$ & $0.922(1)$ & $1.095(1)$ & $0.060(5)$ \\
\hline$c(1)$ & $0.577(2)$ & $0.893(2)$ & $0.866(2)$ & $0.056(7)$ \\
\hline$C(2)$ & $0.891(2)$ & $1.142(1)$ & $0.890(1)$ & $0.037(6)$ \\
\hline $\mathrm{C}(3)$ & $0.491(2)$ & $1.126(1)$ & $0.822(1)$ & $0.036(6)$ \\
\hline$C(4)$ & $0.589(2)$ & $1.128(1)$ & $0.762(1)$ & $0.041(6)$ \\
\hline$\alpha(5)$ & $0.673(2)$ & $1.211(1)$ & $0.778(1)$ & $0.039(6)$ \\
\hline$C(6)$ & $0.622(2)$ & $1.253(1)$ & $0.842(1)$ & $0.044(6)$ \\
\hline$C(7)$ & $0.508(2)$ & $1.204(1)$ & $0.871(1)$ & $0.041(6)$ \\
\hline$C(3 a)$ & $0.364(2)$ & $1.062(2)$ & $0.823(1)$ & $0.052(7)$ \\
\hline$C(4 a)$ & $0.602(2)$ & $1.064(2)$ & $0.694(1)$ & $0.056(7)$ \\
\hline$c(5 a)$ & $0.783(3)$ & $1.245(2)$ & $0.726(1)$ & $0.074(8)$ \\
\hline$C(6 a)$ & $0.675(3)$ & $1.348(1)$ & $0.875(2)$ & $0.081(9)$ \\
\hline$C(7 a)$ & $0.411(2)$ & $1.225(2)$ & $0.935(1)$ & $0.059(8)$ \\
\hline$C(8)$ & $0.987(2)$ & $0.899(1)$ & $0.899(1)$ & $0.032(5)$ \\
\hline$C(9)$ & $0.961(2)$ & $0.910(1)$ & $0.821(1)$ & $0.031(6)$ \\
\hline$C(10)$ & $0.865(2)$ & $0.841(1)$ & $0.796(1)$ & $0.029(5)$ \\
\hline$C(11)$ & $0.839(2)$ & $0.782(1)$ & $0.863(1)$ & $0.029(5)$ \\
\hline$C(12)$ & $0.915(2)$ & $0.815(1)$ & $0.925(1)$ & $0.041(6)$ \\
\hline$C(8 a)$ & $1.093(2)$ & $0.951(2)$ & $0.949(1)$ & $0.049(7)$ \\
\hline$C(9 a)$ & $1.033(2)$ & $0.982(1)$ & $0,768(1)$ & $0.041(6)$ \\
\hline$C(10 a)$ & $0.811(2)$ & $0.824(2)$ & $0.715(1)$ & $0.052(7)$ \\
\hline$C(11 a)$ & $0.756(2)$ & $0.690(1)$ & $0.858(1)$ & $0.040(8)$ \\
\hline$C(12 a)$ & $0.921(2)$ & $0.770(2)$ & $1.004(1)$ & $0.054(7)$ \\
\hline
\end{tabular}

${ }^{a} U_{\text {equir }}=1 / 3$ Spur $\dot{U}$.

(Fig. 1). Wie in $1 \mathrm{~b}$ wird durch das Selenatom die $\mathrm{Rh}-\mathrm{Rh}-$ Bindung geringfügig aufgeweitet, relativ klein ist der $\mathrm{Rh}-\mathrm{Se}-\mathrm{Rh}$-Winkel. Verhältnismässig lang, gemessen an den Kovalenzradien von $\mathrm{S}$ und Se, ist der Abstand Se-S (2.301(6) $\AA$ ) im $\mathrm{SeSO}_{3}$-Liganden. Dieser ist auch sterisch anspruchsvoller als die einfache Se-Brücke, was sich im Vergleich mit $\mathbf{l b}$ in einer Abweichung der $\mathrm{Se}-\mathrm{Rh}-\mathrm{CO}$-Winkel von 90 auf $98.6(8)$ bzw. $85.1(6)^{\circ}$ und einer leichten Verdrillung der $\mathrm{Rh}-\mathrm{Cp}$-Vektoren äussert.

\section{Solvolyse von 1a,b zu den 48-e Clustern $\mathrm{Cp}^{\star}{ }_{3} \mathrm{Rh}_{3}\left(\mu_{3}-\mathrm{CO}\right)\left(\mu_{3}-\mathrm{E}\right)(\mathrm{E}=\mathrm{S}, \mathrm{Se})$}

Die THF-Lösungen der Komplexe 1a,b reagieren bei Raumtemperatur langsam zu den violetten Clustern 4a,b ab (Gl. 2). Deren dreikerniger Charakter wird durch Elementaranalysen und FD-Massenspektren bestätigt. Optimale Ausbeuten im Bereich von $22 \%$ werden für beide Cluster nach $22 \mathrm{~h}$ Reaktionsdauer erhalten. Der zu 4a,b homologe Oxo-Komplex $\mathrm{Cp}^{\star}{ }_{3} \mathrm{Rh}_{3}\left(\mu_{3}-\mathrm{CO}\right)\left(\mu_{3}-\mathrm{O}\right), 4 \mathrm{c}$ ist bereits als 
Tabelle 2

Spektroskopische Daten der Komplexe 3-5 und 7-9

\begin{tabular}{|c|c|c|c|}
\hline & \multicolumn{2}{|l|}{$\mathrm{IR}\left(\mathrm{cm}^{-1}, \mathrm{KBr}\right)$} & \multirow{2}{*}{$\begin{array}{l}{ }^{1} \mathrm{H}-\mathrm{NMR}^{a} \\
\left(\delta\left(\mathrm{CH}_{3}\right)\right)\end{array}$} \\
\hline & $v(\mathrm{CO})$ & $\nu(\mathrm{SO})$ & \\
\hline 3 & $2000^{b}$ & $1239,1230,593$ & 2.05 \\
\hline $4 a$ & 1646 & & 1.84 \\
\hline $4 b$ & 1640 & & 1.84 \\
\hline $4 e^{\circ}$ & 1664 & & 1.83 \\
\hline 5 & & & 1.72 \\
\hline 7 & & & 1.92 \\
\hline 8 & & & $1.85,1.66$ \\
\hline 9 & $1813,1771,1658$ & & 1.73 \\
\hline
\end{tabular}

${ }^{a}$ Varian EM-360L Spektrometer $\left(33^{\circ} \mathrm{C}\right)$; $\mathrm{CDCl}_{3}$-Lösung, i-TMS. ${ }^{b} \mathrm{CH}_{2} \mathrm{Cl}_{2} \cdot{ }^{c}$ Die Werte sind praktisch identisch mit denen aus Ref. 6.

Nebenprodukt (ca. 5\% Ausbeute) bei der nach [6] vollzogenen Synthese von $\mathrm{Cp}_{2}^{\star}{ }_{2} \mathrm{Rh}_{2}(\mathrm{CO})_{2}$ aus $\mathrm{Cp}^{\star} \mathrm{Rh}(\mathrm{CO})_{2}$ und $\mathrm{Me}_{3} \mathrm{NO}$ beschrieben worden. Die $\nu(\mathrm{CO})$ Frequenzen in den IR-Spektren von $\mathbf{4 a - c}$ sind charakteristisch für eine symmetrische CO-Dreifachbrücke, sie werden durch das Heteroatom nur unwesentlich in ihrer Lage beeinflusst (Tab. 2). Praktisch identisch für alle drei Cluster sind die ${ }^{1} \mathrm{H}$-NMR-Spektren, die jeweils nur ein $\mathrm{CH}_{3}$-Resonanzsignal aufweisen.

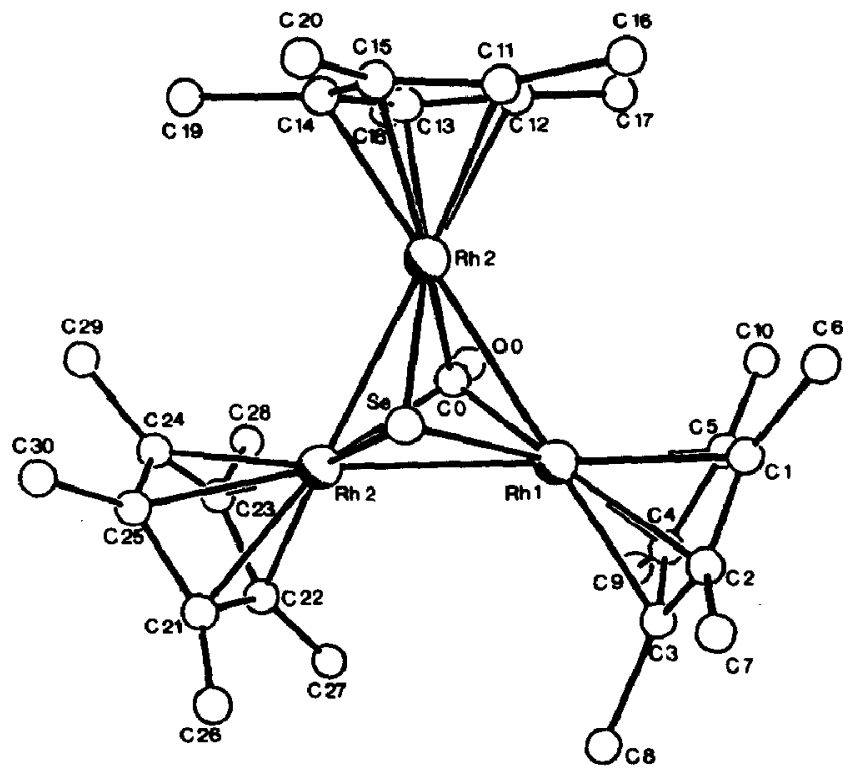

Fig. 2. Struktur von $\mathrm{Cp}^{\star}{ }_{3} \mathrm{Rh}_{3}\left(\mu_{3}\right.$-CO)( $\mu_{3}$-Se) (4b) im Kristall. Ausgewählte Bindungslängen $(\AA)$ und -winkel $\left({ }^{\circ}\right)$ : $\operatorname{Rh}(1)-\operatorname{Rh}(2)$ 2.705(2), $\operatorname{Rh}(1)-\operatorname{Rh}(3)$ 2.710(2), Rh(1)-Se 2.383(3), Rh(1)-C(0) 2.11(1), $\mathrm{Rh}(2)-\operatorname{Rh}(3)$ 2.713(2), Rh(2)-Se 2.375(2), Rh(2)-C(0) 2.13(2), Rh(3)-Se 2.381(2), Rh(3)-C(0) 2.08(2), $\mathrm{C}(0)-\mathrm{O}(0)$ 1.17(2); $\mathrm{Rh}(2)-\mathrm{Rh}(1)-\mathrm{Rh}(3)$ 60.1(1), $\mathrm{Se}-\mathrm{Rh}(1)-\mathrm{C}(0)$ 90.7(5), $\mathrm{Rh}(1)-\mathrm{Se}-\mathrm{Rh}(2)$ 69.3(1), Rh(1)$\mathrm{C}(0)-\mathrm{Rh}(2) 79.4(5)$. 
$\mathbf{1} \mathbf{a , b} \underset{20^{\circ} \mathrm{C} 22 \mathrm{~h}}{\mathrm{THF}} \mathrm{CP}^{*}-\mathrm{R}_{\mathrm{C}}$

\section{$(4 a, b)$}

In Übereinstimmung mit den spektroskopischen Daten befindet sich das Ergebnis einer an Einkristallen von $\mathbf{4 b}$ durchgeführten Röntgenstrukturanalyse (Tab. 3). Zentraler Bestandteil des Moleküls ist eine trigonale Bipyramide, die Rh-Atome bilden ein nahezu gleichseitiges Dreieck (Fig. 2). Entsprechend streuen die Rh-RhAbstände nur wenig um $2.71 \AA$, und es liegen $60^{\circ}$-Winkel an den Rh-Atomen vor.

Tabelle 3

Atomkoordinaten und Temperaturfaktoren von $\mathrm{CP}_{3}{ }_{3} \mathrm{Rh}\left(\mu_{3}-\mathrm{CO}\right)\left(\mu_{3}-\mathrm{Se}\right)(\mathbf{4 b})$

\begin{tabular}{|c|c|c|c|c|}
\hline Atom & $x$ & $y$ & $z$ & $U$ \\
\hline $\operatorname{Rh}(1)$ & $0.1930(2)$ & $0.0749(1)$ & $0.2583(1)$ & $0.028(1)^{a}$ \\
\hline $\operatorname{Rh}(2)$ & $0.1344(2)$ & $0.2755(1)$ & $0.1668(1)$ & $0.032(1)^{a}$ \\
\hline $\operatorname{Rh}(3)$ & $0.1764(2)$ & $0.3080(1)$ & $0.3097(1)$ & $0.029(1)^{a}$ \\
\hline $\mathrm{Se}$ & $0.3924(2)$ & $0.1808(2)$ & $0.2196(1)$ & $0.036(1)^{a}$ \\
\hline$C(0)$ & $-0.008(2)$ & $0.251(1)$ & $0.266(1)$ & $0.035(7)^{a}$ \\
\hline $\mathrm{O}(0)$ & $0.845(1)$ & $0.276(1)$ & $0.282(1)$ & $0.040(5)^{a}$ \\
\hline$C(1)$ & $0.222(2)$ & $-0.115(2)$ & $0.213(1)$ & $0.048(8)^{a}$ \\
\hline$C(2)$ & $0.335(2)$ & $-0.131(2)$ & $0.266(1)$ & $0.057(9)^{a}$ \\
\hline$C(3)$ & $0.235(3)$ & $-0.085(2)$ & $0.335(1)$ & $0.060(10)^{a}$ \\
\hline$C(4)$ & $0.065(2)$ & $-0.043(2)$ & $0.321(1)$ & $0.054(9)^{a}$ \\
\hline$C(5)$ & $0.064(2)$ & $-0.066(2)$ & $0.244(1)$ & $0.046(8)^{a}$ \\
\hline$C(6)$ & $0.286(3)$ & $-0.163(2)$ & $0.136(1)$ & $0.092(8)$ \\
\hline$C(7)$ & $0.527(3)$ & $-0.193(2)$ & $0.260(1)$ & $0.109(9)$ \\
\hline $\mathrm{C}(8)$ & $0.297(3)$ & $-0.087(2)$ & $0.415(1)$ & $0.097(9)$ \\
\hline$C(9)$ & $-0.097(3)$ & $0.011(2)$ & $0.380(1)$ & $0.120(10)$ \\
\hline$C(10)$ & $-0.106(3)$ & $-0.046(2)$ & $0.214(1)$ & $0.096(8)$ \\
\hline $\mathrm{C}(11)$ & $0.151(3)$ & $0.249(2)$ & $0.047(1)$ & $0.063(9)^{a}$ \\
\hline$C(12)$ & $-0.023(4)$ & $0.282(3)$ & $0.080(1)$ & $0.078(14)^{a}$ \\
\hline$C(13)$ & $-0.066(3)$ & $0.406(2)$ & $0.110(1)$ & $0.090(11)^{a}$ \\
\hline$C(14)$ & $0.082(6)$ & $0.443(3)$ & $0.094(2)$ & $0.174(28)^{a}$ \\
\hline$C(15)$ & $0.197(5)$ & $0.342(5)$ & $0.059(2)$ & $0.175(30)^{a}$ \\
\hline$C(16)$ & $0.240(4)$ & $0.127(3)$ & $0.001(1)$ & $0.14(1)$ \\
\hline$C(17)$ & $-0.146(3)$ & $0.204(3)$ & $0.076(1)$ & $0.12(1)$ \\
\hline$C(18)$ & $-0.248(4)$ & $0.475(3)$ & $0.148(2)$ & $0.17(1)$ \\
\hline$C(19)$ & $0.053(4)$ & $0.586(3)$ & $0.122(2)$ & $0.19(2)$ \\
\hline$C(20)$ & $0.371(5)$ & $0.363(3)$ & $0.039(2)$ & $0.22(2)$ \\
\hline$C(21)$ & $0.263(4)$ & $0.352(3)$ & $0.412(2)$ & $0.159(18)^{a}$ \\
\hline $\mathrm{C}(22)$ & $0.102(5)$ & $0.346(2)$ & $0.423(2)$ & $0.150(23)^{\circ}$ \\
\hline$C(23)$ & $0.010(3)$ & $0.444(2)$ & $0.394(1)$ & $0.105(14)^{a}$ \\
\hline $\mathrm{C}(24)$ & $0.093(4)$ & $0.503(2)$ & $0.356(1)$ & $0.107(15)^{a}$ \\
\hline$C(25)$ & $0.247(4)$ & $0.449(3)$ & $0.362(2)$ & $0.124(19)^{a}$ \\
\hline$C(26)$ & $0.441(6)$ & $0.273(4)$ & $0.434(2)$ & $0.26(2)$ \\
\hline $\mathrm{C}(27)$ & $0.027(5)$ & $0.280(4)$ & $0.480(2)$ & $0.29(3)$ \\
\hline $\mathrm{C}(28)$ & $-0.194(5)$ & $0.499(4)$ & $0.391(2)$ & $0.22(2)$ \\
\hline $\mathrm{C}(29)$ & $0.016(5)$ & $0.629(3)$ & $0.317(2)$ & $0.23(2)$ \\
\hline$C(30)$ & $0.388(5)$ & $0.511(4)$ & $0.319(2)$ & $0.26(2)$ \\
\hline
\end{tabular}

${ }^{a} U_{\text {equiv }}=1 / 3$ Spur $\tilde{U}$. 
Die Pyramidenspitzen werden von je einem nahezu symmetrisch angeordneten CObzw. Se-Liganden gebildet.

4a,b sind die ersten strukturell charakterisierten $\mathrm{Rh}$-Vertreter in der Klasse der triangularen 48-e-Cluster $\mathrm{Cp}_{3} \mathrm{M}_{3}\left(\mu_{3}-\mathrm{CO}\right)\left(\mu_{3}-\mathrm{E}\right)(\mathrm{E}=\mathrm{O}, \mathrm{S}, \mathrm{Se})$, von denen bisher nur $\left(\mathrm{C}_{5} \mathrm{H}_{5}\right)$ Co-Derivate bekannt sind [7]. Ihre Bildung belegt erneut die Labilität des $\mathrm{Rh}_{2} \mathrm{E}$-Grundgerüsts der Komplexe 1a,b und deutet auf die zumindest vorübergehende Existenz mehr oder weniger reaktiver Einkernspezies, wie z.B. $\mathrm{Cp}^{\star} \mathrm{Rh}(\mathrm{CO}) \mathrm{E}$ oder $\mathrm{Cp}^{\star} \mathrm{Rh}(\mathrm{CO})$. Derartige Überlegungen werden durch IR-spektroskopische Untersuchungen der Reaktionslösungen gestützt, die Anhaltspunkte für $\mathrm{CO}$-haltige, jedoch sehr labile und daher nicht isolierbare Zwischenstufen bieten [8].

\section{Decarbonylierung von 2 zu dem Heterocuban $\mathrm{Cp}_{4}{ }_{4} \mathrm{Rh}_{4} \mathrm{Se}_{4}$ und Ausweitung der Reaktion auf homologe Co-haltige Komplexe}

Bereits in den FD-Massenspektren von 2 fiel die aussergewöhnlich hohe Bereitschaft zur Abspaltung der CO-Liganden und zur Dimerisierung des verbleibenden Molekülfragments auf [1b]. Diese Reaktion liess sich nunmehr auch präparativ in guten Ausbeuten durch Erwärmen einer Toluollösung von 2 auf $70^{\circ} \mathrm{C}$ nachvollziehen (Gl. 3). Der Einbau von Co anstelle von Rh in den Komplex 2 bewirkt unter massenspektroskopischen Bedingungen eine Verringerung der Dimerisierungstendenz, so dass lediglich $\mathrm{CO}$ abgespalten wird [1b]. Diese Beobachtung deutet auf die mögliche Existenz der dimeren Komplexe 6 unter Rückbildung der M-M-Doppelbindung $\left[9^{*}\right]$. Im präparativen Massstab reagieren jedoch $\mathrm{Cp}^{\star}{ }_{2} \mathrm{MCo}-$ $(\mathrm{CO})_{2}(\mathrm{M}=\mathrm{Co}, \mathrm{Rh})$ unter ansonsten gleichen Bedingungen wie in $\mathrm{Gl} .3 \mathrm{zu}$ den vierkernigen Produkten $\mathrm{Cp}^{\star}{ }_{4} \mathrm{Co}_{4} \mathrm{Se}_{4}$ (7) und $\mathrm{Cp}^{\star}{ }_{4} \mathrm{Co}_{2} \mathrm{Rh}_{2} \mathrm{Se}_{4}$ (8). Auf Grund der ${ }^{1}$ H-NMR-Spektren von 5, 7 und 8 kann eine hochsymmetrische Molekülstruktur angenommen werden, die beiden Methylresonanzen im Spektrum von 8 lassen sich auf die unterschiedliche Natur der beiden Metallatome zurückführen (Tab. 2). Carbonylierungsversuche (100 bar CO, 24 h) führen bei keinem Komplex zu einer Reaktion.

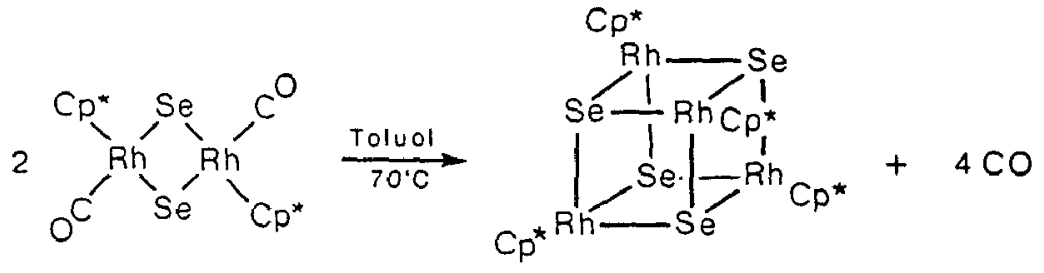

(2)

(5)

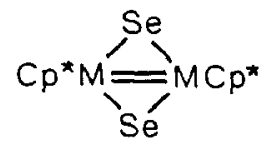

$\left(M=C_{0}, R h\right)$

(6)

\footnotetext{
* Die Literaturnummer mit einem Sternchen deutet eine Bemerkung in der Literaturliste an.
} 
Diese Ergebnisse stehen in Übereinstimmung mit einer cubanartigen Struktur, die für den zu 5, 7 und 8 analogen Schwefelcluster $\left(\mathrm{C}_{5} \mathrm{H}_{5}\right)_{4} \mathrm{Co}_{4} \mathrm{~S}_{4}$ bereits verifiziert worden ist: Der Kern dieses Moleküls besteht aus einem leicht verzerrten $\mathrm{Co}_{4} \mathrm{~S}_{4}$ Würfel mit vernachlässigbaren $\mathbf{M}-\mathbf{M}$-Wechselwirkungen [10]. Isoelektronische Cluster mit Selenliganden, wie z.B. $\mathrm{Fe}_{4}(\mathrm{CO})_{12}\left(\mu_{3}-\mathrm{Se}\right)_{4}[11]$ sind relativ selten [12*]. Das Bindungsgerüst dieser Cluster enthält 72 Valenzelektronen, wobei sämtliche bindenden und antibindenden Energieniveaus des einbeschriebenen $\mathbf{M}_{4}$-Kerns besetzt sind [13].

Isolierung und strukturelle Charakterisierung von $\mathrm{Cp}^{\star}{ }_{3} \mathrm{Rh}_{3}\left(\mu_{3}-\mathrm{CO}\right)\left(\mu_{2}-\mathrm{CO}\right)_{2}(9)$

Als Ausgangsmaterial zur Synthese von 1 und 2 wurde das $\mathrm{Rh}=\mathrm{Rh}$-Dimere $\mathrm{Cp}_{2}{ }_{2} \mathrm{Rh}_{2}(\mu-\mathrm{CO})_{2}$ eingesetzt. Dies wurde nach der von Herrmann et al. entwickelten Decarbonylierung von $\mathrm{Cp}^{\star} \mathrm{Rh}(\mathrm{CO})_{2}$ mittels $\mathrm{Me}_{3} \mathrm{NO}$ dargestellt [6], wobei Ausbeuten um $83 \%$ erzielt wurden. Parallel zur Originalvorschrift [6] gelang uns der Nachweis (Tab. 2) des zu 4a,b homologen Oxoclusters $\mathrm{Cp}_{3}^{\star} \mathrm{Rh}_{3}(\mu-\mathrm{CO})\left(\mu_{3}-\mathrm{O}\right)(\mathbf{4 c})$ der in ca. 6\% Ausbeute entsteht. Neu ist die Existenz eines weiteren Nebenprodukts (4.5\% Ausbeute), dem auf Grund analytischer Untersuchungen (Tab. 5) die Zusammensetzung $\mathrm{CP}^{\star}{ }_{3} \mathrm{Rh}_{3}(\mathrm{CO})_{3}$ (9) zukommt. Laut IR-Spektrum handelt es sich bei 9 um einen ausschliesslich carbonylverbrückten Cluster, die Lage der drei (CO)-Fragmente ist mit der Präsenz von zwei unterschiedlichen Brückentypen vereinbar (Tab. 2). Der Brückencharakter des $\mu_{3}$-CO-Liganden bleibt auch in Lösung (THF, $\mathrm{CH}_{2} \mathrm{Cl}_{2}$, Toluol) erhalten [14].

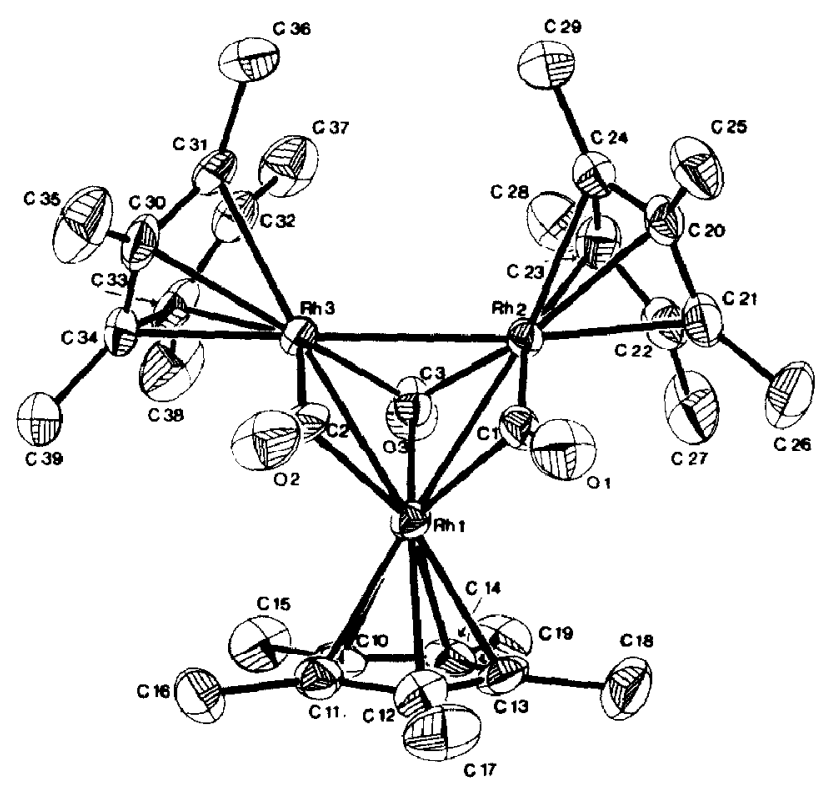

Fig. 3. Struktur von $\mathrm{Cp}^{\star}{ }_{3} \mathrm{Rh}_{3}\left(\mu_{3}-\mathrm{CO}\right)\left(\mu_{2}-\mathrm{CO}\right)_{2}(9)$ im Kristall. Ausgewählte Bindungslängen $(\AA)$ und -winkel $\left({ }^{\circ}\right): \operatorname{Rh}(1)-\operatorname{Rh}(2) 2.678(1), \operatorname{Rh}(1)-\operatorname{Rh}(3) 2.675(1), \operatorname{Rh}(2)-\operatorname{Rh}(3) 2.792(1), \operatorname{Rh}(1)-C(1) 2.077(6)$, $\mathrm{Rh}(2)-\mathrm{C}(1)$ 1.879(7), $\mathrm{Rh}(1)-\mathrm{C}(2)$ 2.101(7), $\mathrm{Rh}(3)-\mathrm{C}(2)$ 1.909(7), $\mathrm{Rh}(1)-\mathrm{C}(3) 2.156(7), \mathrm{Rh}(2)-\mathrm{C}(3)$ 2.009(6), $\mathrm{Rh}(3)-\mathrm{C}(3)$ 2.028(7); $\mathrm{Rh}(2)-\mathrm{Rh}(1)-\mathrm{Rh}(3)$ 62.9(0), $\mathrm{Rh}(1)-\mathrm{Rh}(2)-\mathrm{Rh}(3) 58.5(0), \mathrm{Rh}(1)-\mathrm{C}(1)-$ $\mathrm{O}(1)$ 128.6(5), $\mathrm{Rh}(2)-\mathrm{C}(1)-\mathrm{O}(1)$ 145.9(5), $\mathrm{Rh}(1)-\mathrm{C}(2)-\mathrm{O}(2)$ 129.2(6), $\mathrm{Rh}(3)-\mathrm{C}(2)-\mathrm{O}(2) 147.1(6)$. 
Tabelle 4

Atomkoordinaten und Temperaturfaktoren von $\mathrm{Cp}_{3}{ }_{3} \mathrm{Rh}_{3}\left(\mu_{3}-\mathrm{CO}\right)\left(\mu_{2}-\mathrm{CO}\right)_{2}(9)$

\begin{tabular}{|c|c|c|c|c|}
\hline Atom & $x$ & $y$ & $z$ & $U^{a}$ \\
\hline $\mathbf{R h}(\mathbf{1})$ & $0.20230(4)$ & $0.59542(4)$ & $0.75889(3)$ & $0.0250(2)$ \\
\hline $\mathbf{R h}(2)$ & $0.26964(4)$ & $0.46076(3)$ & $0.86011(3)$ & $0.0256(2)$ \\
\hline $\operatorname{Rh}(3)$ & $0.34419(4)$ & $0.49980(4)$ & $0.70650(3)$ & $0.0264(2)$ \\
\hline$c(1)$ & $0.2837(5)$ & $0.5766(4)$ & $0.8879(4)$ & $0.03(12)$ \\
\hline$O(1)$ & $0.3107(4)$ & $0.6287(3)$ & $0.9462(3)$ & $0.050(2)$ \\
\hline$C(2)$ & $0.3549(5)$ & $0.6133(5)$ & $0.7504(5)$ & $0.035(3)$ \\
\hline$O(2)$ & $0.4055(4)$ & $0.6746(3)$ & $0.7654(4)$ & $0.052(2)$ \\
\hline$C(3)$ & $0.2072(5)$ & $0.4622(5)$ & $0.7291(4)$ & $0,033(3)$ \\
\hline$O(3)$ & $0.1415(4)$ & $0.4179(3)$ & $0.6841(3)$ & $0.041(2)$ \\
\hline $\mathrm{C}(\mathbf{1 0})$ & $0.0772(5)$ & $0.6416(5)$ & $0.6443(5)$ & $0.038(3)$ \\
\hline $\mathrm{C}(11)$ & $0.1443(5)$ & $0.7089(4)$ & $0.6797(5)$ & $0.034(3)$ \\
\hline$C(12)$ & $0.1357(5)$ & $0.7241(5)$ & $0.7681(5)$ & $0.034(3)$ \\
\hline $\mathrm{C}(13)$ & $0.0702(5)$ & $0.6627(5)$ & $0.7912(5)$ & $0.039(3)$ \\
\hline C(14) & $0.0302(5)$ & $0.6110(5)$ & $0.7125(4)$ & $0.037(3)$ \\
\hline$C(15)$ & $0.0611(6)$ & $0.6066(6)$ & $0.5491(4)$ & $0.056(3)$ \\
\hline$C(16)$ & $0.2042(6)$ & $0.7635(5)$ & $0.6253(5)$ & $0.048(31)$ \\
\hline$C(17)$ & $0.1842(6)$ & $0.7951(5)$ & $0.8272(5)$ & $0.058(4)$ \\
\hline $\mathrm{C}(18)$ & $0.0348(6)$ & $0.6568(6)$ & $0.8798(5)$ & $0.061(4)$ \\
\hline$C(19)$ & $-0.0469(5)$ & $0.5383(5)$ & $0.7051(6)$ & $0.059(3)$ \\
\hline$C(20)$ & $0.3331(5)$ & $0.4046(5)$ & $0.9965(4)$ & $0.033(2)$ \\
\hline $\mathrm{C}(21)$ & $0.2298(6)$ & $0.4250(5)$ & $0.9887(4)$ & $0.040(3)$ \\
\hline$C(22)$ & $0.1736(6)$ & $0.3687(5)$ & $0.9232(5)$ & $0.046(3)$ \\
\hline $\mathrm{C}(23)$ & $0.2414(6)$ & $0.3221(5)$ & $0.8848(5)$ & $0.043(3)$ \\
\hline$C(24)$ & $0.3420(6)$ & $0.3439(5)$ & $0.9301(5)$ & $0.037(3)$ \\
\hline$C(25)$ & $0.4180(5)$ & $0.4409(5)$ & $1.0650(5)$ & $0.048(3)$ \\
\hline$C(26)$ & $0.1873(6)$ & $0.4831(6)$ & $1.0518(5)$ & $0.062(4)$ \\
\hline $\mathrm{C}(27)$ & $0.0547(6)$ & $0.3640(7)$ & $0.8962(6)$ & $0.077(4)$ \\
\hline$C(28)$ & $0.2106(7)$ & $0.2542(5)$ & $0.8182(6)$ & $0.064(4)$ \\
\hline$C(29)$ & $0.4357(6)$ & $0.2967(5)$ & $0.9221(6)$ & $0.058(4)$ \\
\hline$C(30)$ & $0.4835(5)$ & $0.5074(5)$ & $0.6433(4)$ & $0.042(3)$ \\
\hline$C(31)$ & $0.4799(6)$ & $0.4254(5)$ & $0.6808(5)$ & $0.038(3)$ \\
\hline$C(32)$ & $0.3904(5)$ & $0.3842(5)$ & $0.6360(5)$ & $0.040(3)$ \\
\hline$C(33)$ & $0.3419(6)$ & $0.4397(6)$ & $0.5695(5)$ & $0.047(3)$ \\
\hline$C(34)$ & $0.3933(6)$ & $0.5175(5)$ & $0.5756(5)$ & $0.041(3)$ \\
\hline$C(35)$ & $0.5676(6)$ & $0.5715(6)$ & $0.6660(6)$ & $0.066(4)$ \\
\hline $\mathrm{C}(36)$ & $0.5647(6)$ & $0.3854(6)$ & $0.7470(5)$ & $0.059(4)$ \\
\hline$C(37)$ & $0.3649(7)$ & $0.2938(5)$ & $0.6456(6)$ & $0.065(4)$ \\
\hline$C(38)$ & $0.2459(6)$ & $0.4192(7)$ & $0.5030(5)$ & $0.081(5)$ \\
\hline$C(39)$ & $0.3747(7)$ & $0.5942(6)$ & $0.5169(5)$ & $0.076(4)$ \\
\hline
\end{tabular}

${ }^{a} U_{\text {equiv }}=1 / 3$ Spur $\tilde{U}$.

Da bisher lediglich $\mathrm{Cp}_{3} \mathrm{Co}_{3}(\mathrm{CO})_{3}$-Cluster strukturell charakterisiert worden sind $[14,15]$, wurde von 9 eine Röntgenstrukturanalyse angefertigt (Tab. 4). Wie aus Fig. 3 hervorgeht, ist das Molekül durch ein gleichschenkliges $R_{h}$-Dreieck charakterisiert. Der Abstand $R h(2)-R h(3)(2.792)(1) \AA$ ) ist um ca. $0.115 \AA$ länger als die beiden anderen $\mathbf{R h}-\mathbf{R h}$-Bindungen. Die Innenwinkel des Dreiecks weichen deutlich von $60^{\circ} \mathrm{ab}$. Über dem Metall-Dreieck sitzt die $\left(\mu_{3} \cdot \mathrm{CO}\right)$-Gruppe, allerdings nicht mehr genau im Zentrum, wie bei 4 b (Fig. 1), sondern mehr gegen die Bindung $R h(2)-R h(3)$ hin orientiert. Die kantenverknüpfenden CO-Liganden sind ebenfalls leicht asymmetrisch verbrückend angeordnet, was sich an den Abständen Rh(2)-C(1) 
(ca. $10 \%$ kürzer als $\mathrm{Rh}(1)-\mathrm{C}(1))$ bzw. $\mathrm{Rh}(3)-\mathrm{C}(2)$ (ca. $10 \%$ kürzer als $\mathrm{Rh}(1)-\mathrm{C}(2))$ ablesen lässt.

\section{Experimenteller Teil}

Einzelheiten bezüglich Arbeitstechnik und Reagentien sind Lit. $1 \mathrm{~b}$ zu entnehmen. Farben, Ausbeuten und analytische Daten sind in Tab. 5, röntgenographische Daten in Tab. 6 zusammengefasst.

Darstellung von $\mathrm{Cp}^{\star}{ }_{2} \mathrm{Rh}_{2}(\mathrm{CO})_{2}\left(\mu-\mathrm{SeSO}_{3}\right)$ (3)

Ein Gemisch aus $306 \mathrm{mg}(0.50 \mathrm{mmol}) \mathbf{1 b}, 80 \mathrm{mg}(0.50 \mathrm{mmol})$ Pyridin $\mathrm{SO}_{3}[16], 20$ $\mathrm{ml}$ Acetonitril und $60 \mathrm{ml}$ Toluol wird $16 \mathrm{~h}$ bei Raumtemperatur gerührt. Nach dem Entfernen des Lösungsmittelgemischs nimmt man den Rückstand in $15 \mathrm{ml}$ Toluol auf, filtriert die Suspension und wäscht mit $20 \mathrm{ml}$ Toluol nach. Im Filtrat können Spuren von $\mathrm{Cp}^{\star}{ }_{3} \mathrm{Rh}_{3}\left(\mu_{3}-\mathrm{CO}\right)\left(\mu_{3}-\mathrm{Se}\right)(4 \mathrm{~b})$ nachgewiesen werden. Der schwarzviolette Rückstand wird in $5 \mathrm{ml} \mathrm{CH} \mathrm{Cl}_{2}$ aufgenommen, auf $\mathrm{Al}_{2} \mathrm{O}_{3}$ (Akt. II-III, Säule $4 \times 3$ $\mathrm{cm}$ ) aufgetragen und mit Ethanol eluiert. Analysenreine Kristalle werden durch Umkristallisation aus $\mathrm{CH}_{2} \mathrm{Cl}_{2} / \mathrm{Hexan} 1 / 1$ bei $-25^{\circ} \mathrm{C}$ erhalten.

\section{Darstellung von $C p^{\star}{ }_{3} R h_{3}\left(\mu_{3}-C O\right)\left(\mu_{3}-E\right)(4 a, b)$}

Eine Lösung von $0.3 \mathrm{mmol} 1 \mathrm{a}$ bzw. $1 \mathrm{~b}$ in $50 \mathrm{ml}$ THF wird $22 \mathrm{~h}$ bei Raumtemperatur gerührt. Nach dem Entfernen des Lösungsmittels nimmt man den Rückstand in $10 \mathrm{ml}$ Toluol auf und chromatographiert an $\mathrm{Al}_{2} \mathrm{O}_{3}$ (Säule $16 \times 3 \mathrm{~cm}$ ). 1a wird mit Toluol, 1b mit Toluol/Ether $5 / 1$ als jeweils violette Zone eluiert. Schwarzviolette Kristalle von 1a,b erhält man durch Umkristallisation aus Pentan bei $-25^{\circ} \mathrm{C}$.

Tabelle 5

Farben, Ausbeuten und Analysenwerte der Komplexe 3-5, 7 und 8

\begin{tabular}{|c|c|c|c|c|c|c|}
\hline \multirow[t]{2}{*}{ Summenformel } & \multirow[t]{2}{*}{ Farbe } & \multirow{2}{*}{$\begin{array}{l}\text { Ausbeute } \\
(\mathscr{\%})\end{array}$} & \multirow{2}{*}{$\begin{array}{l}\text { Molgew. }{ }^{a} \\
\text { (Gef. (ber.)) }\end{array}$} & \multicolumn{3}{|c|}{ Analysen (Gef. (ber.) (官)) } \\
\hline & & & & $\mathrm{C}$ & $\mathbf{H}$ & $\mathrm{Se}$ \\
\hline $\begin{array}{l}\mathrm{C}_{22} \mathrm{H}_{30} \mathrm{Rh}_{2} \mathrm{O}_{5} \mathrm{SSe} \\
\text { (3) }\end{array}$ & $\begin{array}{l}\text { dunkel- } \\
\text { violett }\end{array}$ & 82 & $\begin{array}{r}b \\
(691.3)\end{array}$ & $\begin{array}{c}38.08 \\
(38.22)\end{array}$ & $\begin{array}{c}4.49 \\
(4.37)\end{array}$ & \\
\hline $\begin{array}{l}\mathrm{C}_{31} \mathrm{H}_{45} \mathrm{Rh}_{3} \mathrm{OS} \\
(4 \mathrm{a})\end{array}$ & $\begin{array}{l}\text { dunkel- } \\
\text { violett }\end{array}$ & 22 & $\begin{array}{l}774 \\
(774.5)\end{array}$ & $\begin{array}{c}48.47 \\
(48.08)\end{array}$ & $\begin{array}{c}5.80 \\
(5.86)\end{array}$ & \\
\hline$\underset{(4 b)}{\mathrm{C}_{31} \mathrm{H}_{45} \mathrm{Rh}_{3} \mathrm{OSe}}$ & $\begin{array}{l}\text { dunkel- } \\
\text { violett }\end{array}$ & 23 & $\begin{array}{c}822 \\
(821.4)\end{array}$ & $\begin{array}{c}45.42 \\
(45.33)\end{array}$ & $\begin{array}{c}5.51 \\
(5.52)\end{array}$ & $\begin{array}{c}9.57 \\
(9.61)\end{array}$ \\
\hline $\begin{array}{l}\mathrm{C}_{40} \mathrm{H}_{60} \mathrm{Rh}_{4} \mathrm{Se}_{4} \\
\text { (5) }\end{array}$ & rotbraun & 60 & $\begin{array}{c}1272^{\circ} \\
(1268.4)\end{array}$ & $\begin{array}{c}38.07 \\
(37.87)\end{array}$ & $\begin{array}{c}4.75 \\
(4.77)\end{array}$ & \\
\hline $\begin{array}{l}\mathrm{C}_{40} \mathrm{H}_{60} \mathrm{Co}_{4} \mathrm{Se}_{4} \\
(7)\end{array}$ & $\begin{array}{l}\text { dunkel- } \\
\text { grau }\end{array}$ & 72 & $\begin{array}{l}1096^{\circ} \\
(1092.4)\end{array}$ & $\begin{array}{c}44.61 \\
(43.98)\end{array}$ & $\begin{array}{c}5.71 \\
(5.54)\end{array}$ & \\
\hline $\begin{array}{l}\mathrm{C}_{40} \mathrm{H}_{60} \mathrm{Co}_{2} \mathrm{Rh}_{2} \mathrm{Se}_{4} \\
\quad(8)\end{array}$ & $\begin{array}{l}\text { dunkel- } \\
\text { braun }\end{array}$ & 63 & $\begin{array}{l}1184^{c} \\
(1180.4)\end{array}$ & $\begin{array}{c}41.38 \\
(40.70)\end{array}$ & $\begin{array}{c}5.19 \\
(5.12)\end{array}$ & \\
\hline $\begin{array}{l}\mathrm{C}_{33} \mathrm{H}_{45} \mathrm{Rh}_{3} \mathrm{O}_{3} \\
\quad(9)\end{array}$ & rotbraun & 4.5 & $\begin{array}{l}798 \\
(798.5)\end{array}$ & $\begin{array}{c}49.80 \\
(49.64)\end{array}$ & $\begin{array}{c}5.57 \\
(5.68)\end{array}$ & \\
\hline
\end{tabular}

${ }^{a}$ Felddesorptionsmassenspektren (Gerät Varian 311A. Toluollösung). ${ }^{b}$ Bei Fadenheizungen um $20 \mathrm{~mA}$ werden die Clusterionen $\mathrm{Cp}_{3}^{\star} \mathrm{Rh}_{3}(\mathrm{CO}) \mathrm{Se}^{+}\left(m / e\right.$ 822) und $\mathrm{Cp}_{3}^{\star} \mathrm{Rh}_{3} \mathrm{Se}_{2}^{+}$( $m / e$ 874) beobachtet. ${ }^{c}$ Bzgl. ${ }^{80} \mathrm{Se}$. 
Tabelle 6

Röntgenographische Daten der Komplexe 3, 4b und 9

\begin{tabular}{|c|c|c|c|}
\hline & $\mathrm{Cp}_{2}{ }_{2} \mathrm{Rh}_{2}(\mathrm{CO})_{2}\left(\mathrm{SeSO}_{3}\right)(3)$ & $\mathrm{Cp}_{3}{ }_{3} \mathrm{Rh}_{3}(\mathrm{CO}) \mathrm{Se}(4 \mathrm{~b})$ & $\mathrm{Cp}_{3}{ }_{3} \mathrm{Rh}_{3}(\mathrm{CO})_{3}(9)$ \\
\hline Farbe & Tiefpurpur & Schwarzviolett & Rotbraun \\
\hline Gitterkonstanten $a(\AA)$ & $9.857(3)$ & $8.382(4)$ & $13.444(9)$ \\
\hline$b(\AA)$ & $14.265(3)$ & $11.062(5)$ & $15.790(7)$ \\
\hline$c(\AA)$ & $17.736(5)$ & $18.241(8)$ & $15.249(9)$ \\
\hline$\alpha\left(0^{\circ}\right)$ & 90 & $89.27(4)$ & 90 \\
\hline$\beta\left(^{\circ}\right)$ & 90 & $83.62(4)$ & $101.33(5)$ \\
\hline$\gamma\left({ }^{\circ}\right)$ & 90 & $70.64(4)$ & 90 \\
\hline Zellvolumen $V\left(\AA^{3}\right)$ & 2498 & 1585.4 & 3173.98 \\
\hline Besetzungszahl $Z$ & 4 & 2 & 4 \\
\hline $\begin{array}{l}\text { Dichte (röntgeno- } \\
\text { graphisch) }\left(\mathrm{g} / \mathrm{cm}^{3}\right)\end{array}$ & 1.71 & 1.72 & 1.67 \\
\hline $\begin{array}{l}\text { Raumgruppe } \\
\text { Kristallgrosse }\left(\mathrm{mm}^{3}\right)\end{array}$ & $\begin{array}{l}P 2_{1} 2_{1} 2_{1} \\
0.30 \times 0.20 \times 0.15\end{array}$ & $\begin{array}{l}P \overline{1} \\
0.3 \times 0.35 \times 0.4\end{array}$ & $\begin{array}{l}C_{2 h}^{5}-P 21 / c \\
0.27 \times 0.38 \times 0.42\end{array}$ \\
\hline Diffraktometer & AED II & AED II & AED II \\
\hline $\begin{array}{l}\text { Strahlung } \\
\text { Messbereich }\left({ }^{\circ}\right)\end{array}$ & $\begin{array}{l}\text { Mo- } K_{\alpha} \\
3<2 \theta<45\end{array}$ & $\begin{array}{l}\text { Mo- } K_{\alpha} \\
3<2 \theta<55\end{array}$ & $\begin{array}{l}\text { Mo- } K_{\alpha} \\
3<2 \theta<59\end{array}$ \\
\hline Gemessene Reflexe & 1292 & 3794 & 5580 \\
\hline Unabhängige Reflexe & $1178(I>\sigma(I))$ & $3329(I>2.5 \sigma(I))$ & $4806(I>2.5 \sigma(I))$ \\
\hline Strukturbestimmung & Patterson/Fourier & Patterson/Fourier & Patterson/Fourier \\
\hline$R$ & 0.042 & 0.067 & 0.046 \\
\hline$R_{\mathbf{w}}$ & 0.036 & 0.056 & 0.037 \\
\hline
\end{tabular}

Allgemeine Arbeitsvorschrift zur Darstellung der Komplexe 5, 7 und 8

Ca. $0.25 \mathrm{mmol}$ der Komplexe $\mathrm{Cp}^{\star}{ }_{2} \mathrm{MM}^{\prime}(\mathrm{CO})_{2} \mathrm{Se}_{2}$ werden in $70 \mathrm{ml}$ Toluol $\left(\mathbf{M}=\mathbf{M}^{\prime}=\mathrm{Rh} ; \mathbf{M}=\mathrm{Co}, \mathbf{M}^{\prime}=\mathrm{Rh}\right)$ oder $70 \mathrm{ml} \mathrm{THF}\left(\mathbf{M}=\mathbf{M}^{\prime}=\mathbf{C o}\right)$ gelöst und 50 min bei $70^{\circ} \mathrm{C}$ gerührt. Das Solvens wird anschliessend eingeengt bzw. abgezogen, und die Rohprodukte werden in $\mathrm{Al}_{2} \mathrm{O}_{3}$ (Säule $12 \times 3 \mathrm{~cm}$ ) mit Toluol $(5,8)$ oder $\mathrm{Et}_{2} \mathrm{O}$ (7) chromatographiert.

Isolierung von $C p^{\star}{ }_{3} R h_{3}\left(\mu_{3}-\mathrm{CO}\right)(\mu-\mathrm{CO})_{2}(9)$ aus der Synthese von $C p^{\star}{ }_{2} R h_{2}(\mathrm{CO})_{2}$ nach [6]

Die Lösung von $2.23 \mathrm{~g}(7.58 \mathrm{mmol}) \mathrm{Cp}^{\star} \mathrm{Rh}(\mathrm{CO})_{2}$ und $0.78 \mathrm{~g}(10.38 \mathrm{mmol})$ $\mathrm{Me}_{3} \mathrm{NO}$ in $150 \mathrm{ml}$ Aceton wird $2 \mathrm{~h}$ am Sieden gehalten. Nach dem Entfernen des Lösungsmittels wird der violettschwarze Rückstand in $20 \mathrm{ml}$ Toluol aufgenommen und an $\mathrm{SiO}_{2}$ (Säule $28 \times 4 \mathrm{~cm}$ ) chromatographiert. Mit Toluol eluiert man zunächst eine orange Zone $\left(\mathrm{Cp}^{\star} \mathrm{Rh}(\mathrm{CO})_{2}\right)$ und dann mit Toluol/Ether 10/1 eine dunkelblaue Zone $\left(\mathrm{CP}_{2}^{\star}{ }_{2} \mathrm{Rh}_{2}(\mathrm{CO})_{2}\right)$. Mit Ether/Aceton $1 / 1$ lässt sich eine weitere, dunkelviolette Zone eluieren. Diese Zone liefert durch wiederholte Chromatographie $\left(\mathrm{SiO}_{2}\right.$, Säule $7 \times 3 \mathrm{~cm}$ ) ca. $90 \mathrm{mg}$ an rotbraunem 9 (Eluens Toluol/Ether 1/1) sowie ca. $115 \mathrm{mg}$ an rotviolettem 4c (Eluens Ether/Aceton 2/1). Dunkelrotbraune Kristalle von 9 erhält man durch Umkristallisieren aus Toluol/Pentan 2/1.

\section{Literatur}

1 (a) W.A. Herrmann, C. Bauer und J. Weichmann, Chem. Ber., 117 (1984) 1271; W.A. Herrmann und J. Weichmann, Organomet. Synth., 3 (1986) 287; (b) H. Brunner, N. Janietz, W. Meier, E. Herdtweck, 
W.A. Herrmann, O. Serhadli und M.L. Ziegler, J. Organomet. Chem., 347 (1988) 237; H. Brunner, N. Janietz, J. Wachter, B. Nuber und M.L. Ziegler, ibid., 367 (1989) 197.

2 H. Brunner, N. Janietz, W. Meier, B. Nuber, J. Wachter und M.L. Ziegler, Angew. Chem., 100 (1988) 717; Angew. Chem. Int. Fd. Fngl., 27 (1988) 708.

3 H. Brunner, W. Meier, B. Nuber, J. Wachter und M.L. Ziegler, Angew. Chem., 98 (1986) 907; Angew. Chem. Int. Ed. Engl., 25 (1986) 908.

4 G.J. Kubas, H.J. Wasserman und R.R. Ryan, Organometallics, 4 (1985) 419; G.J. Kubas, R.R. Ryan, K.A. Kubat-Martin und E. Larson, J. Am. Chem. Soc., 111 (1989) 7823.

5 H. Brunner, U. Klement, J. Pfauntsch und J. Wachter, Angew. Chem., 99 (1987) 268; Angew. Chem. Int. Ed. Engl., 26 (1987) 230; H. Brunner, H. Kauermann, J. Pfauntsch, J. Wachter, J.H. Enemark und C.G. Young, J. Organomet. Chem., 331 (1987) 45; K.A. Kubat-Martin, G.J. Kubas und R.R. Ryan, Organometallics, 8 (1989) 1910.

6 W.A. Herrmann, J. Plank, C. Bauer, M.L. Ziegler, E. Guggolz und R.R. Alt, Z. Anorg. Allg. Chem., 487 (1982) 85; W.A. Herrmann, J. Plank und C. Bauer, Organomet. Synth., 3 (1986) 48.

7 V.A. Uchtman und L.F. Dahl, J. Am. Chem. Soc., 91 (1969) 3763; S. Otsuka, A. Nakamura und T. Yoshida, Inorg. Chem., 7 (1968) 261; P.D. Frisch und L.F. Dahl, J. Am. Chem. Soc., 94 (1972) 5082; W.L. Olson, A.M. Stacy und L.F. Dahl, ibid., 108 (1986) 7646.

8 N. Janietz, Dissertation Universität Regensburg, 1989.

9 Die einzige bisher fassbare Verbindung dieses Typs, jedoch mit Oxobrücken, ist $\mathrm{Cp}_{2}^{\star} \mathrm{Ir}_{2} \mathrm{O}_{2}: \mathrm{W}$.D. McGhee, T. Foo, F.J. Hollander und R.C. Bergman, J. Am. Chem. Soc., 110 (1988) 8543.

10 G.L. Simon und L.F. Dahl, J. Am. Chem. Soc., 95 (1973) 2164.

11 L.L. Nelson, F.Y.-K. Lo, A.D. Rae und L.F. Dahl, J. Organomet. Chem., 225 (1982) 309.

$12 \mathrm{Co}_{4}\left(\mathrm{PPh}_{3}\right)_{4}\left(\mu_{3}-\mathrm{Se}\right)_{4}$ ist dagegen als 60-e-Cluster mit einbeschriebenem $\mathrm{Co}_{4}$-Tetraeder aufzufassen: $\mathrm{D}$. Fenske, J. Ohmer und J. Hachgenei, Angew. Chem., 97 (1985) 993; Angew. Chem. Int. Ed., 24 (1985) 993.

13 C.T.-W. Cho, F.Y.-K. Lo und L.F. Dahl, J. Am. Chem. Soc., 95 (1973) 2164.

14 F.A. Cotton und J.D. Jameson, J. Am. Chem. Soc., 98 (1976) 1273; R.J. Lawson und J.R. Shapley, ibid., 98 (1976) 7433; R.J. Lawson und J.R. Shapley, Inorg. Chem., 17 (1978) 772.

15 O.S. Mills und E.F. Paulus, Chem. Commun., 1966, 815; L.M. Cirjak, J.-S. Huang. Z.-H. Zhu und L.F. Dahl, J. Am. Chem. Soc., 102 (1980) 6623; C.E. Barnes und M.R. Dial, Organometallics, 7 (1988) 782 .

16 H.H. Sisler und L.F. Audrieth, Inorg. Synth., II (1946) 173. 